\title{
Detector Module Design, Construction and Performance for the LHCb SciFi Tracker
}

\author{
Robert Ekelhof ${ }^{* *}$ \\ TU Dortmund \\ E-mail: robert.ekelhofetu-dortmund.de
}

The Scintillating Fibre (SciFi) Tracker for the LHCb Upgrade (CERN/LHCC 2014-001; LHCb TDR 15) is based on $2.5 \mathrm{~m}$ long multi-layered ribbons from $10000 \mathrm{~km}$ of scintillating fibre over 12 planes covering $350 \mathrm{~m}^{2}$. The planes are separated into modular detectors, each with cooled silicon photomultiplier (SiPM) arrays for photo-readout. In this contribution, we will present the construction and performance of this novel detector, including the intricacies of scintillating fibre ribbon production, constructing precision detector planes with a rigid and light module design, and the integration of the readout components for this detector. The complexities and issues regarding this active part of the SciFi Tracker will be emphasised along with the current solutions and measured performances.

Technology and Instrumentation in Particle Physics 2014,

2-6 June, 2014

Amsterdam, the Netherlands

\footnotetext{
* Speaker.

$\dagger$ on behalf of the LHCb SciFi Tracker Group
} 


\section{Overview}

The SciFi Tracker is part of the LHCb Upgrade to be installed in 2018/19 ${ }^{1}$. It consists of twelve planes of $6 \mathrm{~m}$ width and $5 \mathrm{~m}$ height (see Fig. 1). The detector is made of scintillating fibres of $250 \mu \mathrm{m}$ diameter, read out by multichannel Silicon-Photomultipliers (SiPM). The SiPMs are cooled to $-40^{\circ} \mathrm{C}$ to reduce the radiation induced dark counts. The single modules are $52 \mathrm{~cm}$ wide and cover the full height of the $\mathrm{LHCb}$ acceptance of $5 \mathrm{~m}$. Every detector plane consists of 5-6 layers of fibres to guarantee a sufficiant light yield. The fine read-out pitch of $250 \mu \mathrm{m}$ (SiPM pitch) leads to low occupancies and good hit resolution $(<100 \mu \mathrm{m})$.

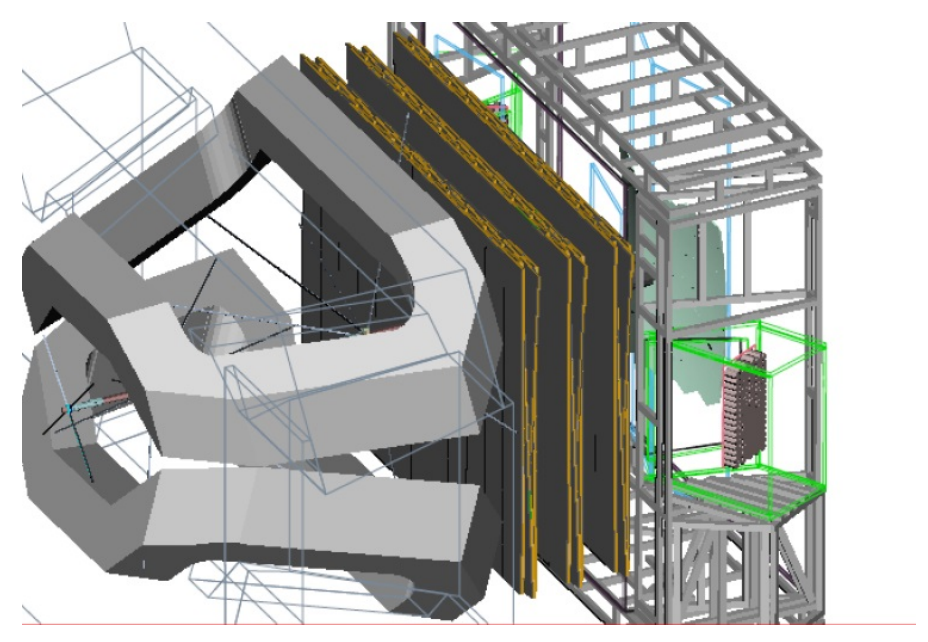

Figure 1: The three stations of the scintillating fibre tracker shown between the dipole magnet on the left and RICH2 on the right. [1]

\section{Fibre Mats}

The scintillating fibres are wound on a wheel with a thread. The thread guarantees the correct pitch. At the end of the layer the fibre is cut and placed at the starting edge. From the second layer on, the fibres of the previous layer serve as thread. TiO2-loaded glue is placed between the layers. After curing the mat is cut perpendicular to the fibres and taken of the wheel to be flattened. To ensure precise positioning of the fibre mat on the support panel, alignment pins are automatically produced during winding. In order to achieve a robust and flat surface the raw mats are precisely embedded in extra glue. They are then cut parallel to the fibres to obtain the correct geometry. The center edge of the modules (opposite to the SiPMs) are mirrored to increase the light yield. Aluminised mylar film has proven to provide high reflectivity $(>80 \%)$ in combination with the best operability.

\section{Panels}

The panel is built as a sandwich of $0.2 \mathrm{~mm}$ carbon-fibre skins seperated by two $20 \mathrm{~mm}$ layers

\footnotetext{
${ }^{1}$ All the information in this note is taken from the LHCb Tracker Upgrade Technical Design Report [1].
} 
of light core material (Divinycell F40) on either side of the scintillating fibre mats. One panel spans the size of a complete module ( $5 \mathrm{~m}$ high, $0.52 \mathrm{~m}$ wide) and carries four fibre mats side by side on the upper half, and four on the lower half. On the upper and lower edges the light core material is replaced by polycarbonate pieces which serve as connection to the Read-out Box and to the mounting frames. They ensure the alignment between the frames, the panels, the fibres and the SiPMs.

\section{Read-out Box}

The Read-out Box (ROB) is situated outside the acceptance, it hosts (see Fig. 2) the outer end of the fibre mats, the SiPMs (16 arrays of 128 channels), the cooling of the SiPMs and the frontend electronics boards. In this small volume around the SiPMs, the thermal insulation and gas- and

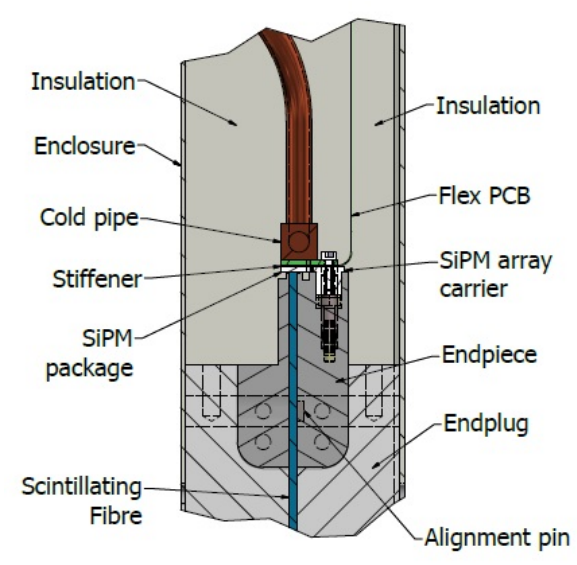

Figure 2: The Schematic view of the cold volume within the Read-out Box. [1]

light-tightness have to be guaranteed. All the components need to stay above the dew point. Inside the ROB this is insured by flushing with dry air. The outside is kept at higher temperatures by the help of the insulation and the use of heat spreaders. The ROB will provide several service features, like temperature sensors, light injection system to calibrate the SiPM gain. Access will be possible separately for the warm electronics and the cold SiPM part.

\section{Performance}

Several tests have been performed with different components (no complete module is available yet). The optical analysis of the fibre mat cross-section (see Fig. 3) showed that the RMS of the fibre pitch is $9-16 \mu \mathrm{m}$ (1st-5th layer). The light yield of mats in test-beam and with radioactive sources was measured. The attenuation length was $>4 \mathrm{~m}$, with good uniformity across the fibre mat. The straightness of the fibres after aligning to a support with the help of the alignment pins was measured. The RMS of the centre fibre along the length was found to be $25 \mu \mathrm{m}$. The stiffness of the support panels was tested. The Young's modulus was measured to be $1.73 \mathrm{GPa}$ and therefore better than the one of the Outer Tracker modules (1.24 GPa). 


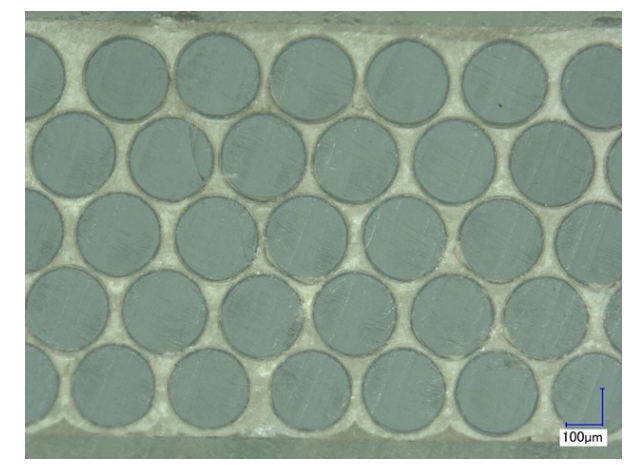

Figure 3: A photograph of a cross-section of a fibre mat.

\section{Summary}

Based on the gained experience, a functional module design can be produced. Several components are already available as prototypes and were successfully tested. Fibre mats were produced in serial-production-like conditions and were tested for their light yield. They will be mounted on the support panels for a test beam at the end of this year.

In parallel, mechanical Read-out Box prototypes were tested including cooling. The next stage of development will be the inclusion of SiPMs in the ROB to be connected to fibres.

The mechanical design regarding the cooling of the SiPMs, thermal expansion and preventing condensation and frost build-up inside and outside the ROB have been identified as challenges. These aspects will be addressed in the future Engineering Design Report. The Technical Design Report of the LHCb Tracker is available via the CERN document server [1].

\section{References}

[1] LHCb Tracker Upgrade Technical Design Report, LHCb Collaboration, 2014, LHCB-TDR-015, CERN-LHCC-2014-001 